\title{
Theoretical description of deeply virtual Compton scattering off ${ }^{3} \mathrm{He}$
}

Received: date / Accepted: date

\begin{abstract}
Recently, coherent deeply virtual Compton scattering (DVCS) off ${ }^{3}$ He nuclei has been proposed to access the neutron generalized parton distributions (GPDs). In Impulse Approximation (IA) studies, it has been shown, in particular, that the sum of the two leading twist, quark helicity conserving GPDs of ${ }^{3} \mathrm{He}, H$ and $E$, at low momentum transfer, is dominated by the neutron contribution, so that ${ }^{3} \mathrm{He}$ is very promising for the extraction of the neutron information. Nevertheless, such an extraction could be not trivial. A technique, able to take into account the nuclear effects included in the IA analysis in the extraction procedure, has been therefore developed. In this work, the IA calculation of the spin dependent GPD $\tilde{H}$ of ${ }^{3} \mathrm{He}$ is presented for the first time. This quantity is found to be largely dominated, at low momentum transfer, by the neutron contribution, which could be extracted using arguments similar to the ones previously proposed for the other GPDs. The known forward limit of the IA calculation of $\tilde{H}$, yielding the polarized parton distributions of ${ }^{3} \mathrm{He}$, is correctly recovered. The knowledge of the GPDs $H, E$ and $\tilde{H}$ of ${ }^{3} \mathrm{He}$ will allow now the evaluation of the cross section asymmetries which are relevant for coherent DVCS off ${ }^{3} \mathrm{He}$ at Jefferson Lab kinematics, an important step towards the planning of possible experiments.
\end{abstract}

Keywords Three body systems · Generalized parton distributions

Initially introduced in Ref. [1], Generalized Parton Distributions (GPDs), among other features, represent a crucial tool to shed light on the so called "Spin Crisis" problem. As a matter of fact, GPDs measurements will allow to access the parton total angular momentum [2]. By subtracting from the latter the helicity quark contribution, measured in other hard processes, the parton orbital angular momentum (OAM) could be then estimated.

Deeply Virtual Compton Scattering (DVCS), i.e. the reaction $e H \longmapsto e^{\prime} H^{\prime} \gamma$ when $Q^{2} \gg M^{2}$ $\left(Q^{2}=-q \cdot q\right.$ is the momentum transfer between the leptons $e$ and $e^{\prime}, \Delta^{2}$ the one between hadrons $H$ and $H^{\prime}$ with momenta $P$ and $P^{\prime}$, and $M$ is the nucleon mass), is considered the cleanest process to access GPDs. Another relevant kinematical variable is the so called skewedness, $\xi=-\Delta^{+} /\left(P^{+}+P^{\prime+}\right)$ 1. Despite severe difficulties to extract GPDs from experiments, data for proton and nuclear targets are being analyzed, see, i.e., Refs. [3; 4]. The measurement of GPDs for nuclei could be crucial to distinguish between different models of nuclear medium modifications of the nucleon structure, an impossible task in the analysis of DIS experiments only (this discussion started in Ref. [5]). As always,

M. Rinaldi

Dipartimento di Fisica, Università degli studi di Perugia and INFN sezione di Perugia, Via A. Pascoli 06100 Perugia, Italy

E-mail: matteo.rinaldi@pg.infn.it

S. Scopetta

Dipartimento di Fisica, Università degli studi di Perugia and INFN sezione di Perugia, Via A. Pascoli 06100 Perugia, Italy

E-mail: sergio.scopetta@pg.infn.it

${ }^{1}$ In this paper, $a^{ \pm}=\left(a^{0} \pm a^{3}\right) / \sqrt{2}$. 

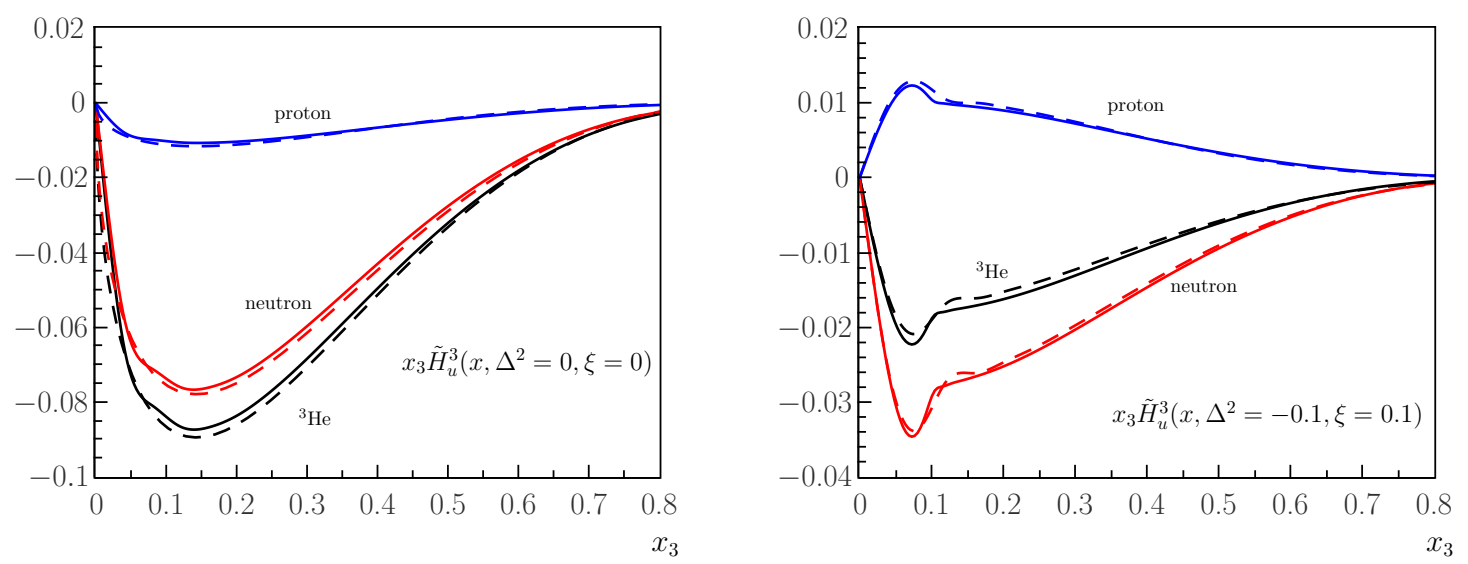

Fig. 1 The $q=u$ flavor GPD $x_{3} \tilde{H}^{3, u}\left(x, \Delta^{2}, \xi\right)$, where $x_{3}=\left(M_{3} / M\right) x$ and $\xi_{3}=\left(M_{3} / M\right) \xi$, shown in the forward limit (left panel) and at $\Delta^{2}=-0.1 \mathrm{GeV}^{2}$ and $\xi_{3}=0.1$ (right panel), together with the neutron and the proton contribution. Solid lines represent the full IA result, Eq. (2), while the dashed ones correspond to the approximation Eq. (6).

the neutron measurement, which requires nuclear targets, is important because it permits, together with the proton one, a flavor decomposition of GPDs. In studying observables related to the neutron polarization, ${ }^{3} \mathrm{He}$ plays a special role, due to its spin structure (see, e.g., Refs. [6; 7]). This is true in particular for GPDs. Indeed, among the light nuclei ${ }^{3} \mathrm{He}$ is the only one for which the quantity $\tilde{G}_{M}^{3, q}\left(x, \Delta^{2}, \xi\right)=H_{q}^{3}\left(x, \Delta^{2}, \xi\right)+E_{q}^{3}\left(x, \Delta^{2}, \xi\right)$, i.e., the sum of its GPDs $H_{q}$ and $E_{q}$, could be dominated by the neutron, being the isoscalar targets ${ }^{2} \mathrm{H}$ and ${ }^{4} \mathrm{He}$ not suitable to this aim, as it has been discussed in Ref. [8]. In the same papers it has been also shown to what extent this fact can be used to extract the neutron information.

The formal treatment of ${ }^{3} \mathrm{He}$ GPDs in Impulse Approximation (IA) can be found in Refs. [9], where, for the GPD $H$ of ${ }^{3} \mathrm{He}, H_{q}^{3}$, a convolution-like equation in terms of the corresponding nucleon quantity has been obtained. The treatment has been later extended to $\tilde{G}_{M}^{3, q}$ (see Ref. [8] for details), and to $\tilde{H}_{q}^{3}$, yielding

$$
\tilde{G}_{M}^{3, q}\left(x, \Delta^{2}, \xi\right)=\sum_{N} \int d E \int d \mathbf{p}\left[P_{+-,+-}^{N}-P_{+-,-+}^{N}\right]\left(\mathbf{p}, \mathbf{p}^{\prime}, E\right) \frac{\xi^{\prime}}{\xi} \tilde{G}_{M}^{N, q}\left(x^{\prime}, \Delta^{2}, \xi^{\prime}\right),
$$

and

$$
\tilde{H}_{q}^{3}\left(x, \Delta^{2}, \xi\right)=\sum_{N} \int d E \int d \mathbf{p}\left[P_{++,++}^{N}-P_{++,--}^{N}\right]\left(\mathbf{p}, \mathbf{p}^{\prime}, E\right) \frac{\xi^{\prime}}{\xi} \tilde{H}_{q}^{N}\left(x^{\prime}, \Delta^{2}, \xi^{\prime}\right),
$$

respectively.

In the last two equations, $x^{\prime}$ and $\xi^{\prime}$ are the variables for the bound nucleon GPDs, $p\left(p^{\prime}=p+\Delta\right)$ is its 4-momentum in the initial (final) state and, eventually, proper components appear of the spin dependent, one body off diagonal spectral function:

$$
P_{S S^{\prime}, s s^{\prime}}^{N}\left(\mathbf{p}, \mathbf{p}^{\prime}, E\right)=\frac{1}{(2 \pi)^{6}} \frac{M \sqrt{M E}}{2} \int d \Omega_{t} \sum_{s_{t}}\left\langle\mathbf{P}^{\prime} S^{\prime} \mid \mathbf{p}^{\prime} s^{\prime}, \mathbf{t} s_{t}\right\rangle_{N}\left\langle\mathbf{p} s, \mathbf{t} s_{t} \mid \mathbf{P} S\right\rangle_{N},
$$

where $S, S^{\prime}\left(s, s^{\prime}\right)$ are the nuclear (nucleon) spin projections in the initial (final) state, respectively, and $E=E_{\min }+E_{R}^{*}$, being $E_{R}^{*}$ the excitation energy of the full interacting two-body recoiling system. The main quantity appearing in the definition Eq. (3) is the intrinsic overlap integral

$$
\left\langle\mathbf{p} s, \mathbf{t} s_{t} \mid \mathbf{P} S\right\rangle_{N}=\int d \mathbf{y} e^{i \mathbf{p} \cdot \mathbf{y}}\left\langle\chi_{N}^{s}, \Psi_{t}^{s_{t}}(\mathbf{x}) \mid \Psi_{3}^{S}(\mathbf{x}, \mathbf{y})\right\rangle
$$


between the wave function of ${ }^{3} \mathrm{He}, \Psi_{3}^{S}$, and the final state, described by two wave functions: i) the eigenfunction $\Psi_{t}^{s_{t}}$, with eigenvalue $E=E_{m i n}+E_{R}^{*}$, of the state $s_{t}$ of the intrinsic Hamiltonian pertaining to the system of two interacting nucleons with relative momentum $\mathbf{t}$, which can be either a bound or a scattering state, and ii) the plane wave representing the nucleon $N$ in IA. For a numerical evaluation of Eqs. (1) and (2), the overlaps, Eq. (4), appearing in Eq. (3) and corresponding to the analysis presented in Ref. [10] in terms of AV18 [11] wave functions [12], have been used. For the nucleonic GPDs, a simple model for $\tilde{G}_{M}^{N, q}$ and $\tilde{H}_{q}^{N}$ [13], properly extended to evaluate also spin dependent GPDs (see Ref. 8] for details), has been used. It is worth noticing that Eq. (2) and the results of its numerical evaluation are presented here for the first time. Since there are no ${ }^{3} \mathrm{He}$ data available, it is possible to verify only a few general GPDs properties, i.e., the forward limit and the first moments. In particular the calculation of $H_{q}^{3}\left(x, \Delta^{2}, \xi\right)$ fulfills these constraints [9]. In the $\tilde{G}_{M}^{3, q}\left(x, \Delta^{2}, \xi\right)$ case, since there is no observable forward limit for $E_{q}^{3}\left(x, \Delta^{2}, \xi\right)$, the only possible check is the first moment: $\sum_{q} \int d x \tilde{G}_{M}^{3, q}\left(x, \Delta^{2}, \xi\right)=G_{M}^{3}\left(\Delta^{2}\right)$; where $G_{M}^{3}\left(\Delta^{2}\right)$ is the magnetic form factor (ff) of ${ }^{3}$ He. The result obtained has been found to be in agreement with previous calculations (e.g. the one-body part of the AV18 calculation presented in Ref. [14]) and, for the values of $\Delta^{2}$ which are relevant for the coherent process under investigation here, i.e., $-\Delta^{2} \leq 0.15 \mathrm{GeV}^{2}$, our results compare well also with the data.

Let us discuss now our calculation of $\tilde{H}_{q}^{3}$, presented here for the first time. First of all, we checked that the forward limit of our expression, Eq. (2), reproduces formally and numerically the formalism obtained in Ref. 7] for polarized DIS off ${ }^{3} \mathrm{He}$. On the other hand, the first moment of $\tilde{H}_{q}^{3}$ is related to the axial form factor of ${ }^{3} \mathrm{He}$, an observable poorly known which does not permit therefore a consistency check. With the comfort of the fulfillment of the forward limit constraint we can now proceed to analyze the proton and neutron contributions to the ${ }^{3} \mathrm{He}$ observable. Since $\tilde{H}_{q}^{3}$ is measured using a polarized target, it should be dominated by the neutron contribution. Let us show now to what extent this feature is obtained and how, thanks to this observation, the neutron information can be extracted.

The results of the numerical evaluation of Eq. (2) are presented in Fig. 1. In the forward limit, the neutron contribution strongly dominates the ${ }^{3}$ He quantity, but increasing $\Delta^{2}$ the proton contribution grows up (see Fig. 1, solid lines in both panels), in particular for the $u$ flavor. It is therefore necessary to introduce a procedure to safely extract the neutron information from ${ }^{3} \mathrm{He}$ data. This can be done by observing that Eq. (2) can be written as

$$
\tilde{H}_{q}^{3}\left(x, \Delta^{2}, \xi\right)=\sum_{N} \int_{x_{3}}^{\frac{M_{A}}{M}} \frac{d z}{z} h_{N}^{3}\left(z, \Delta^{2}, \xi\right) \tilde{H}_{q}^{N}\left(\frac{x}{z}, \Delta^{2}, \frac{\xi}{z},\right)
$$

where $h_{N}^{3}\left(z, \Delta^{2}, \xi\right)$ is a "light cone spin dependent off-forward momentum distribution" which is strongly peaked around $z=1$ close to the forward limit. Therefore, in this region, for $x_{3}=\left(M_{A} / M\right) x \leq$ 0.7 one has:

$$
\begin{aligned}
\tilde{H}_{q}^{3}\left(x, \Delta^{2}, \xi\right) & \simeq \sum_{N} \tilde{H}_{q}^{N}\left(x, \Delta^{2}, \xi\right) \int_{0}^{\frac{M_{A}}{M}} d z h_{N}^{3}\left(z, \Delta^{2}, \xi\right) \\
& =G_{A}^{3, p, p o i n t}\left(\Delta^{2}\right) \tilde{H}_{q}^{p}\left(x, \Delta^{2}, \xi\right)+G_{A}^{3, n, p o i n t}\left(\Delta^{2}\right) \tilde{H}_{q}^{n}\left(x, \Delta^{2}, \xi\right)
\end{aligned}
$$

Here, the axial point like ff, $G_{A}^{3, N, \text { point }}\left(\Delta^{2}\right)=\int_{0}^{\frac{M_{A}}{M}} d z h_{N}^{3}\left(z, \Delta^{2}, \xi\right)$, which would give the nuclear axial $\mathrm{ff}$ if the proton and the neutron were point-like particles, are introduced. These quantities, at small values of $\Delta^{2}$, depend weakly on the potential used in the calculation, so that the theoretical error in their evaluation is small. This can be realized observing that, in the forward limit, they reproduce the so called "effective polarizations" of the protons $\left(p_{p}\right)$ and the neutron $\left(p_{n}\right)$ in ${ }^{3}$ He, whose values are rather similar if evaluated within different nucleon nucleon potentials (see Refs. [6; 7; 10] for a comprehensive discussion). In particular, within the AV18 potential under scrutiny here, the values $p_{n}=0.878$ and $p_{p}=-0.023$ are obtained. Eq. (6) can now be used to extract the neutron contribution from possible sets of data for the proton and for ${ }^{3} \mathrm{He}$ :

$$
\tilde{H}^{n, e x t r}\left(x, \Delta^{2}, \xi\right) \simeq \frac{1}{G_{A}^{3, n, p o i n t}\left(\Delta^{2}\right)}\left\{\tilde{H}^{3}\left(x, \Delta^{2}, \xi\right)-G_{A}^{3, p, p o i n t}\left(\Delta^{2}\right) \tilde{H}^{p}\left(x, \Delta^{2}, \xi\right)\right\} .
$$


The comparison we have done between the free neutron GPDs, used as input in the calculation, and the ones extracted using our calculation for $\tilde{H}^{3}$ and the proton model for $\tilde{H}^{p}$, shows that the procedure works nicely even beyond the forward limit. The only theoretical ingredients are the axial point like ffs, which, as explained above, are under good theoretical control. The procedure works for $x \leq 0.7$, where possible data are expected from JLab.

In closing, we have shown that coherent DVCS off ${ }^{3} \mathrm{He}$ at low momentum transfer $\Delta^{2}$ is an ideal process to access the neutron GPDs; if data were taken at higher $\Delta^{2}$, a relativistic treatment [15] and/or the inclusion of many body currents, beyond the present IA scheme, should be implemented. The next step of this investigation will be the evaluation of cross section asymmetries relevant to DVCS experiments at JLab kinematics, using the obtained theoretical GPDs $H, E$ and $\tilde{H}$ of ${ }^{3} \mathrm{He}$. At the beginning, the leading twist analysis of DVCS for a spin 1/2 target, presented in Ref. [16], will be performed.

This work was supported in part by the Research Infrastructure Integrating Activity Study of Strongly Interacting Matter (acronym HadronPhysic3, Grant Agreement n. 283286) under the Seventh Framework Programme of the European Community.

\section{References}

1. Mueller, D. et al.: Wave functions, evolution equations and evolution kernels from light ray operators of QCD. Fortsch. Phys. 42, 101 (1994);

2. Radyushkin, A. V. Scaling limit of deeply virtual Compton scattering. Phys. Lett. B 380, 417 (1996); Ji,X. -D. Gauge invariant decomposition of nucleon spin and its spin - off . Phys. Rev. Lett. 78, 610 (1997).

3. Mazouz, M. et al. [Jefferson Lab Hall A Collaboration]: Deeply virtual compton scattering off the neutron. Phys. Rev. Lett. 99242501 (2007).

4. Guidal, M: Constraints on the $\tilde{H}$ Generalized Parton Distribution from Deep Virtual Compton Scattering Measured at HERMES. Phys. Lett. B 693, 17 (2010).

5. Berger, E. R., Cano, F., Diehl, M., and Pire, B.: Generalized parton distributions in the deuteron. Phys. Rev. Lett. 87, 142302 (2001).

6. Friar, J. L. et al.: Neutron polarization in polarized He-3 targets. Phys. Rev. C 422310 (1990).

7. Ciofi degli Atti, C. et al.: Nuclear effects in deep inelastic scattering of polarized electrons off polarized He-3 and the neutron spin structure functions. Phys. Rev. C 48968 (1993).

8. Rinaldi, M. and Scopetta, S: Neutron orbital structure from generalized parton distributions of $3 \mathrm{He}$. Phys. Rev. C 85, 062201(R) (2012); Rinaldi, M. and Scopetta, S: Extracting neutron generalized parton distributions from 3He data. Phys. Rev. C 87, 035208 (2013).

9. Scopetta, S: Generalized parton distributions of ${ }^{3}$ He. Phys. Rev. C 70015205 (2004); Scopetta, S: Conventional nuclear effects on generalized parton distributions of trinucleons.Phys. Rev. C 79025207 (2009).

10. Kievsky, A., Pace, E., Salmè, G. and Viviani, M.: Neutron electromagnetic form-factors and inclusive scattering of polarized electrons by polarized He-3 and He-3 targets. Phys. Rev. C 56, 64 (1997).

11. Wiringa, R. B., Stoks V. G. J. and Schiavilla, R.: An Accurate nucleon-nucleon potential with charge independence breaking. Phys. Rev. C 5138 (1995).

12. Kievsky, A., Viviani, M. and Rosati, S.: Study of bound and scattering states of three nucleon systems. Nucl. Phys. A 577511 (1994).

13. Musatov, I. V. and Radyushkin, A. V.: Evolution and models for skewed parton distributions. Phys. Rev. D 61074027 (2000).

14. Marcucci, L. E., Riska, D. O. and Schiavilla, R.: Electromagnetic structure of trinucleons. Phys. Rev. C 58, 3069 (1998).

15. Pace, E, Salmè, G., Scopetta, S., Del Dotto, A. and Rinaldi, M.: Neutron Transverse-Momentum Distributions and Polarized ${ }^{3} \mathrm{He}$ within Light-Front Hamiltonian Dynamics. Few Body Syst. 54, 1079 (2013).

16. Belitsky, A. V., Mueller, D., Niedermeier, L. and Schäfer, A.: Leading twist asymmetries in deeply virtual Compton scattering. Nucl. Phys. B 593, 289 (2001). 